\title{
Stress-Strain Relationships in Yarns Subjected to Rapid Impact Loading: 3. Effect of Wave Propagation
}

\author{
Jack C. Smith, Frank L. McCrackin, and Herbert F. Schiefer
}

\begin{abstract}
The tensile behavior of a Hookean material, elongated by rapid impact at one end has been calculated, using a theory in which wave propagation is considered. As a result of these calculations, limits have been established on the applicability of a simpler theory, discussed in a preceding publication, in which wave propagation was neglected.
\end{abstract}

\section{Introduction}

When a filament of uniform cross section is clamped at each end and rapidly elongated, data for a stress-strain curve can be obtained by measuring the force at one end and the elongation of the whole filament, both as functions of the time. In this case the assumption is made that stresses and strains in the filament are uniform along the length and can be represented by the stresses measured at an end and by the average elongations. This assumption is no longer valid when the rate at which the filament is lengthened approaches in order of magnitude the velocity of propagation of a tension wave along the filament.

In previous work ${ }^{2}$ this effect of wave propagation was disregarded. It is the purpose here to determine how previously obtained results must be reinterpreted as a result of the wave-propagation effect.

\section{Wave Theory}

Consider a filament lying along the negative $x$-axis, with one end at the origin and having the other end fixed at the point $x=-L$. At time $t=0$ and subsequently, the end at $x=0$ is constrained to move with velocity $v_{0}$ in the positive direction. Assume that the tensile behavior is in accordance with Hooke's law. Then, after impact, a strain pulse of magnitude $\epsilon_{0}=v_{0} / c$ is propagated down the filament with a velocity $c$. When the pulse is reflected at the fixed end, the strain is increased to $2 \epsilon_{0}$. A similar increase takes place at each reflection.

This behavior is shown in figure 1, in which the strains at several points along the filament are plotted as functions of the time. The strains are thus seen to vary discontinuously with time. These discontinuous increases in the strain become more pronounced as $v_{0}$ is increased. If we require that $\epsilon_{0}$ be less than 1 percent, in order that wave-propagation effects in most textile fibers may be safely disregarded, we must limit $v_{0}$ to values less than $0.01 c$, which in the case of high-tenacity nylon is approximately $25 \mathrm{~m} / \mathrm{sec}$.

In the present experimental method a filament is terminated at $x=-L$ by a mass $n w$, where $n$ is a number and $w$ is the mass of the filament. At $x=0$ the filament is terminated by a mass so large that its momentum will be practically unchanged by reaction forces from the filament and attached mass. At time $t=0$ the head mass is given a velocity $v_{0}$. The behavior of the system is recorded photographically. ${ }^{3}$ The data of interest are the elongation of the filament and the reaction force applied to the filament at the tail mass, both as functions of time. From these data the experimental stress-strain curve is constructed.

\footnotetext{
1 This work was sponsored by the Office of the Quartermaster General, Department of the Army, and was presented in part at the November 1954 meeting of the Society of Rheology.

${ }^{2}$ Frank L. McCrackin, Herbert F. Schiefer, Jack C. Smith, and Walter K. Stone, Stress-strain relationships in yarns subjected to rapid impact loading: 2. Breaking velocities, strain energies, and theory neglecting wa ve propagation, J. Research NBS 54, 277 (1955) R P2590.

${ }^{3}$ Walter K. Stone, Herbert F. Schiefer, and George Fox, Stress-strain relationships in yarns subjected to rapid impact loading: 1. Equipment, testing procedure, and typical results, J. Research NBS 54, 269 (1955) RP2589.
} 


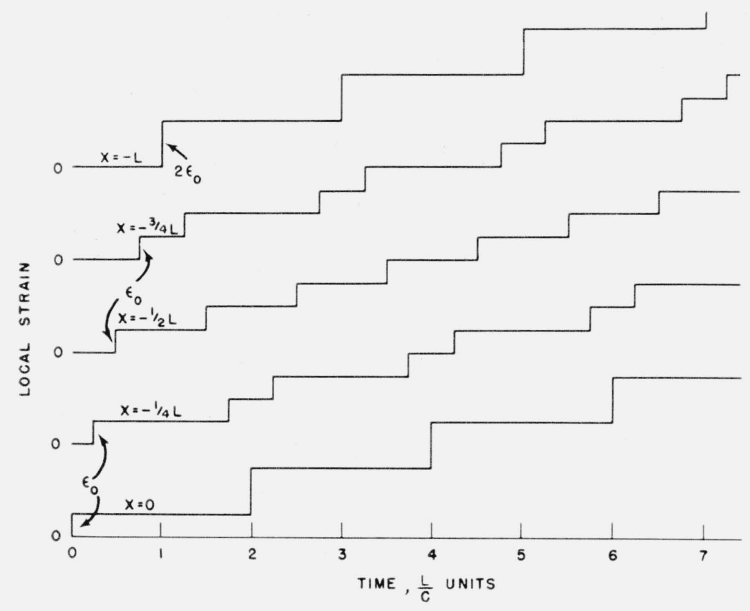

Figure 1. Local strain versus time for a filament clamped at $x=-L$ and rapidly elongated at $x=0$ at velocity $v_{0}$.

The theoretical behavior of this system, when Hooke's law applies, ${ }^{4}$ is most easily calculated by finding suitable solutions of the wave equation

$$
\frac{\partial^{2} u}{\partial t^{2}}=c^{2} \frac{\partial^{2} u}{\partial x^{2}}
$$

In this equation, $t$ is the time variable, and $x$ denotes the position of a cross section of the filament relative to fixed coordinates, when the filament is in the unstrained state; $u(x, t)$ denotes the distance the cross section has moved from its original position at $x$, and $c$ is the velocity of propagation of an elastic-tension wave along the filament. This velocity is given by $c=\sqrt{E / \rho}$, where $E$ is Young's modulus of elasticity for the unstrained filament, and $\rho$ is the density of the unstrained filament material.

These solutions must satisfy the boundary condition at the head:

$$
u(0, t)=v_{0} t
$$

A suitable solution (for the unreflected wave) is given by

$$
\begin{aligned}
u(x, t) & =0 & & \left(t+\frac{x}{c} \leq 0\right) \\
& =v_{0}\left(t+\frac{x}{c}\right) & & \left(t+\frac{x}{c}>0\right) \\
\epsilon(x, t) & =\frac{\partial u}{\partial x}=0 & & \left(t+\frac{x}{c} \leq 0\right) \\
& =\frac{v_{0}}{c} & & \left(t+\frac{x}{c}>0\right),
\end{aligned}
$$

where $u$ represents a wave of velocity $c$ traveling along the filament in the minus $x$-direction, and $\epsilon$ is the local strain, or increase in length per unit length, set up in the filament in the wake of the wave. It should be noted that both $u$ and $\epsilon$ are equal to zero for values of $t<|x| / c$, i. e., no strain or displacement is present at a point in advance of the wave.

When the wave arrives at the end of the filament it is reflected and travels back in the positive direction. The strain between the reflected wave front and the tail mass is represented

4 The stress-strain behavior of most textile fibers may be described by Hooke's law only for small strains. At larger strains, the solutions derived here provide only rough approximations to the behavior of an actual textile fiber but are adequate for the purposes of this theory.

In order to take into account the significant reduction in cross-sectional area of the filament for the finite strains considered here, it is assumed that Hooke's law is obeyed in such a way that the product of Young's modulus and cross-sectional area remains constant. The small effect of mass motion associated with the lateral contraction has been neglected. 


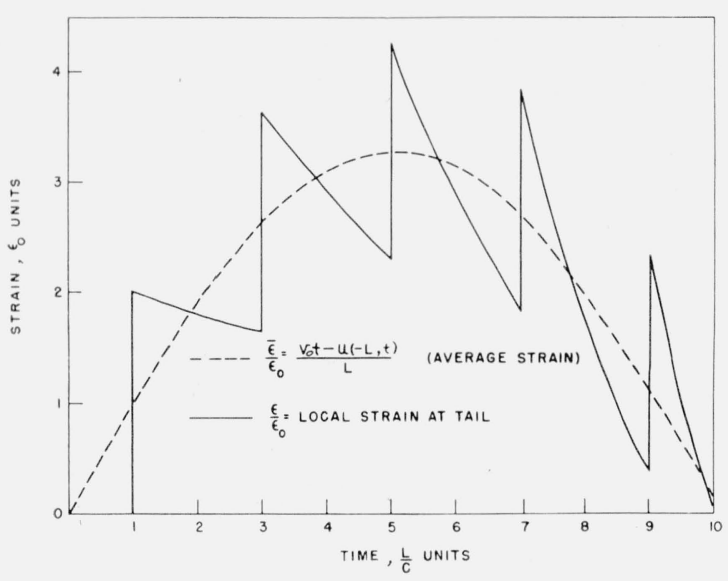

Figure 2. Strain-time curves for a filament loaded at the tail with a mass $10 w$ and rapidly elongated at the head at velocity $v_{0}$.

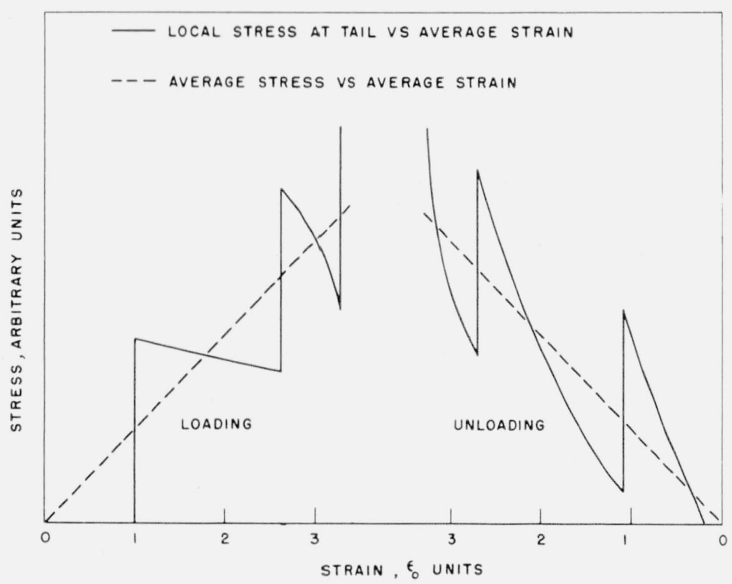

Figure 3. Stress-strain curves for a filament loaded with tail mass $10 \mathrm{w}$ and rapidly elongated at the head at velocity $v_{0}$.

as the sum of the strains caused by the incident and reflected waves. If the tail end of the filament were fixed, this strain would be $2 \epsilon_{0}$. However, as the tail mass can move, this strain, initially $2 \epsilon_{0}$ at reflection, will decrease with time. The rate of decrease depends upon the tail mass and upon the order of the reflection; i. e., whether it is the first or a subsequent reflection. Reflections also occur at the head mass. A different solution of the wave equation is required to describe the state of the filament after each reflection. The first 10 of these solutions are tabulated in appendix 1 .

By using these solutions it is possible to calculate curves of average strain versus time, and local strain versus time. These curves are given in figure 2 for the case where the tail has a mass of $10 w(n=10)$. The curve of local strain, $\epsilon(-L, t)$, versus time is jagged, the decrease in $\epsilon$ between successive reflections becoming greater with each reflection.

As the force at the tail, which is found experimentally from the acceleration of the tail mass, is proportional to the strain at the tail, it is possible to construct a theoretical stress-strain curve of local tail stress (strain) versus average strain. Such a curve is given in figure 3 . Experimental curves are similar to this curve in that they show dips in the vicinity of maximum stress. There is also a delay in the initial rise of stress by the time it takes for the tension wave to be propagated to the tail mass.

\section{Theory, Neglecting Wave Propagation}

A solution, applicable at low testing speeds, can be obtained by disregarding wave propagation along the filament, and assuming the mass of the filament to be concentrated partly at the tail and partly at the head. The resulting solution, derived in appendix 2 , is

$$
\begin{gathered}
u=\epsilon_{0} L\left[\theta-\sqrt{n^{\prime}} \sin \frac{\theta}{\sqrt{n^{\prime}}}\right] \\
{\left[\frac{\epsilon}{\epsilon_{0}}\right]=\sqrt{n^{\prime}} \sin \frac{\theta}{\sqrt{n^{\prime}}}}
\end{gathered}
$$

where $\theta=(c / L) t, \epsilon_{0}=v_{0} / c, n^{\prime}=n+f$, and $f$ is the fraction of the filament mass assumed concentrated at the tail.

It is helpful to think of $\theta$ as time expressed in $L / c$ units. Likewise, one can think of $\left[\epsilon / \epsilon_{0}\right]$ as strain expressed in $\epsilon_{0}$ units. Here $\epsilon_{0}$ and $c$ have only formal significance in order to put the solution in a form analogous to that obtained when wave propagation is considered. 


\section{Comparison of Wave Theory With Theory Neglecting Wave Propagation}

Plots of $\left[\epsilon / \epsilon_{0}\right]$ versus $\theta$ for values of $n^{\prime}=10,10 \frac{1}{2}$, and 11 are shown in figure 4 . Values of $\bar{\epsilon} / \epsilon_{0}$ have also been computed from the wave theory for the case $n=10$, using the formula

$$
\frac{\bar{\epsilon}}{\epsilon_{0}}=\frac{v_{0} t-u(-L, t)}{\epsilon_{0} L}=\theta-\frac{c}{L v_{0}} u(-L, t) .
$$

These points are plotted as circles and are seen to fit best to the curve drawn for $n^{\prime}=10 \frac{1 / 2}{2}$. This indicates that for a mass ratio of 10 , the nonwave theory results agree most closely with those for the wave theory when half the mass of the filament is assumed concentrated at each end $\left(f=\frac{1}{2}\right)$.

According to the nonwave theory, the maximum average strain that can be obtained in a filament is given by

$$
\left[\frac{\epsilon}{\epsilon_{0}}\right]_{\max }=\sqrt{n+f}=\sqrt{n+\frac{1}{2}}
$$

and this strain is obtained when $\theta_{m}=(c / L) t_{m}=(\pi / 2) \sqrt{n+\frac{1}{2}}$ or

$$
t_{m}=\frac{\pi}{2} \frac{L}{c} \sqrt{n+\frac{1}{2}}
$$

This suggests that the maximum average strain $\bar{\epsilon} / \epsilon_{0}$ as computed by the wave theory is approximately equal to $\sqrt{n+\frac{1}{2}}$ and occurs for a value of $\theta_{m}$ approximately equal to $\pi / 2 \sqrt{n+\frac{1}{2}}$. That this is a good approximation is demonstrated in table 1.

According to the nonwave theory (appendix 2)

$$
\log v_{n}=-\frac{1}{2} \log \left(n+\frac{1}{2}\right)+\log \sqrt{\frac{2}{\rho} \int_{0}^{\epsilon_{r}} \sigma d \epsilon},
$$

where $v_{n}$ is the lowest impact velocity to cause rupture in a filament to which a tail mass, $n w$, is attached, $\sigma$ is the tensile stress expressed as force divided by the cross-sectional area of the unstrained filament, and $\boldsymbol{\epsilon}_{\tau}$ is the strain at which rupture occurs.

$$
\begin{aligned}
& \text { TABLE 1. Values of maximum average strain and position of } \\
& \text { maximum average strain as computed by the wave theory } \\
& \text { and by the nonwave theory }
\end{aligned}
$$

\begin{tabular}{|r|c|c|c|c|}
\hline \multirow{2}{*}{$n$} & \multicolumn{2}{|c|}{ Maximum average strain } & \multicolumn{2}{|c|}{ Position } \\
\cline { 2 - 3 } & $\begin{array}{c}\text { Wave theory } \\
\bar{\epsilon} / \epsilon_{0}\end{array}$ & $\begin{array}{c}\text { Nonwave theory } \\
\sqrt{n+\frac{1}{2}}\end{array}$ & $\begin{array}{c}\text { Wave theory } \\
\theta_{m}\end{array}$ & $\begin{array}{c}\text { Nonwave theory } \\
\pi / 2 \sqrt{n+\frac{1}{2}}\end{array}$ \\
\hline & & & & \\
2 & 1.61 & 1.58 & 2.39 & 2.48 \\
5 & 2.39 & 2.34 & 3.59 & 3.68 \\
10 & 3.26 & 3.24 & 5.10 & 5.08 \\
25 & 5.13 & 5.05 & 7.90 & 7.93 \\
\hline
\end{tabular}

If the values of $\boldsymbol{\epsilon}_{\tau}$ and $\sigma$ are independent of the rate of elongation, then $\log \sqrt{(2 / \rho) \int_{0}^{\epsilon_{r}} \sigma d \epsilon}$ will be a constant, and the plot of $\log v_{n}$ versus $\log \left(\mathrm{n}+\frac{1}{2}\right)$ will be a straight line of slope $-\frac{1}{2}{ }^{5}$

\footnotetext{
${ }^{5}$ At high rates of straining, the value of $\epsilon_{\tau}$ for an actual textile fiber is less than that obtained at conventional testing rates. Also the slope of the stress-strain curve is greater at higher rates of straining. However, for rates of straining of the same order of magnitude, these changes are small, and tend to cancel each other out. Consequently, the area under the stress-strain curve, or rupture energy density in the filament, should be essentially constant for rates of straining corresponding to impact velocities near the limiting breaking velocity.
} 
When $\log \left(n+\frac{1}{2}\right)$ is zero, we have the extrapolated velocity

When Hooke's law applies, we have

$$
v_{b}=\sqrt{\frac{2}{\rho} \int_{0}^{\epsilon_{r}} \sigma d \epsilon}
$$

$$
v_{b}=\sqrt{\frac{E}{\rho} \epsilon_{r}^{2}}
$$

According to the wave theory, $\sqrt{E / \rho}$ is the velocity of propagation, $c$, of an elastic tension wave along the filament, and thus

$$
v_{b}=c \epsilon_{r},
$$

the velocity just sufficient to cause immediate rupture at $x=0$.

We call the velocity defined by (10) the "limiting breaking velocity". The concept of a limiting breaking velocity has been discussed, in a previous paper (see footnote 2 ).

As an example, consider a Hookean material for which $\boldsymbol{\epsilon}_{\tau}=0.12$, and $c=2,500 \mathrm{~m} / \mathrm{sec}$.

From (7), for the case of rupture, we have $\epsilon_{r}=\epsilon_{0} \sqrt{n+\frac{1}{2}}=\left(v_{n} / c\right) \sqrt{n+\frac{1}{2}}$ or $v_{n}=\frac{c \epsilon_{r}}{\sqrt{n+\frac{1}{2}}}$.

In figure 5 are plotted values of $v_{n}$ computed from these assumed values of $\epsilon_{\tau}$ and $c$. The result is a straight line giving an extrapolated value of $300 \mathrm{~m} / \mathrm{sec}$ as the limiting breaking velocity.

The breaking velocities have also been calculated on the basis of the more exact theory in which wave motion is considered and Hooke's law is assumed. Average strains are calculated from (6), and the breaking velocities are then computed from the formula

$$
v_{n}=\frac{c \epsilon_{r}}{\left[\frac{\overline{\boldsymbol{\epsilon}}(n)}{\epsilon_{0}}\right]_{\max }} .
$$

These results are also plotted in figure 5. It is seen that these breaking velocities, for a Hookean material of constant $\boldsymbol{\epsilon}_{r}$, are lower (for small $n$ 's) than those predicted by the nonwave theory.

In actuality the strain at a given point along the filament does not change continuously with time but increases suddenly by an amount $\epsilon_{0}$ when one of the strain pulses passes through the point. Rupture then occurs whenever the local strain exceeds the rupture strain.

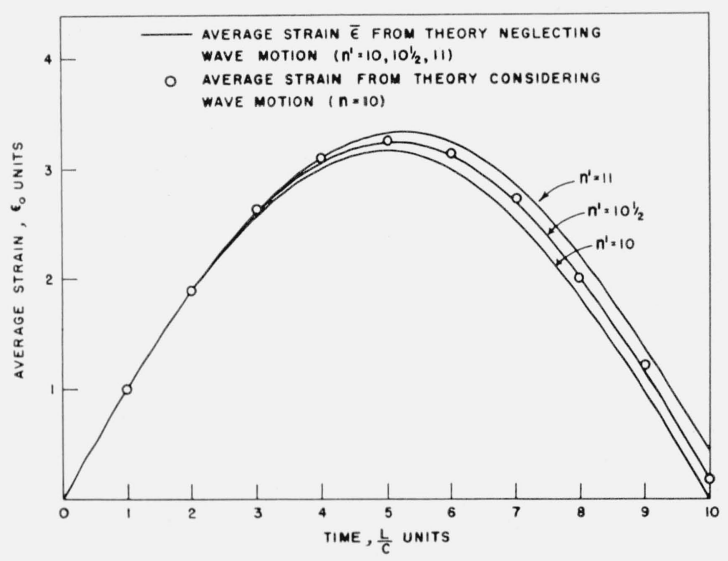

Figure 4. Strain-time curve for filament loaded with tail mass $10 w$, according to the wave theory, and according to the theory neglecting wave propagation.

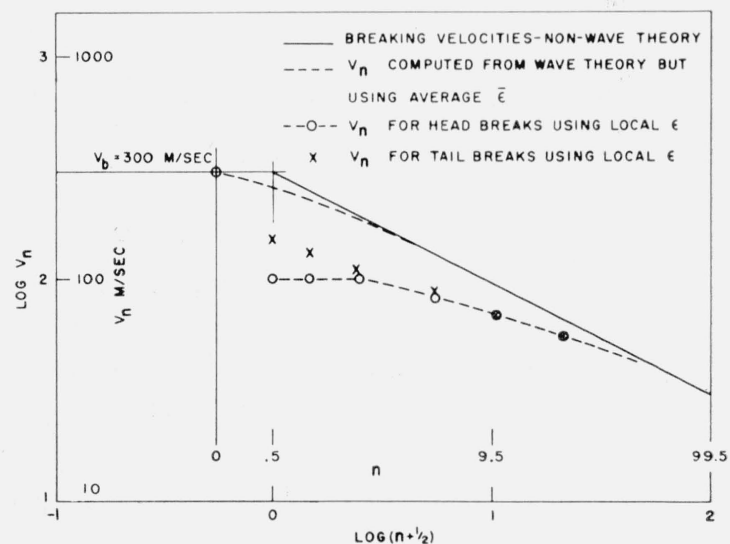

Figure 5. Breaking velocities for a hypothetical specimen, as computed by the wave theory, and by the nonwave theory. 


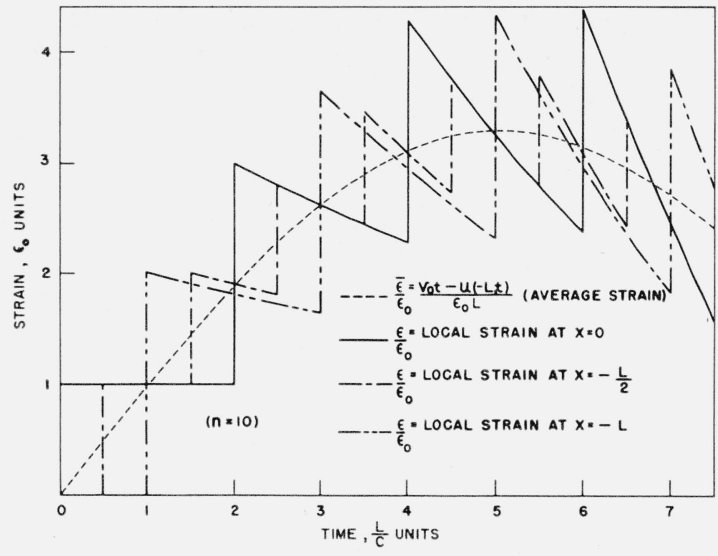

Figure 6.-Local strain-time curves for a filament loaded with a tail-mass $10 \mathrm{w}$.

In figure 6 are plotted local strains at the head, tail, and midpoint for a filament loaded with a tail mass $10 w(n=10)$. Note here that maximum strain occurs at the head on the third reflection $(\theta=6)$. This corresponds to a lowest breaking velocity of $68.6 \mathrm{~m} / \mathrm{sec}$.

Increases in the local strain always occur first upon reflection at either the head or the tail. Increases in the local strain at an intervening point occur later, but these strains never exceed the head or tail strains in magnitude. For this reason, it is to be expected that a uniformly strong filament will break only at the ends.

If the maximum values of local strains $\left[\epsilon / \epsilon_{0}\right]_{\max }$ are substituted into eq 14 , the lowest breaking velocities, $v_{n}$, for head and tail breaks can be computed. These breaking velocities, also plotted in figure 5, are seen to be the lowest of all.

The curves of local strains versus time for an actual material, having approximately the properties chosen for this example, would not be as jagged as those shown in figure 6 for an ideal Hookean material. Thus, the velocities found experimentally for this actual material could be expected to lie between the bounds established in figure 5 by the curve of the velocities computed from average strains in the filament, and the curve of velocities computed from the extreme local strains at the head or tail of the filament. The breaking velocities computed by any of the methods considered are the same when $n$ is greater than 50 . It is thus possible, in this example, to determine a limiting breaking velocity by extrapolation of experimental data taken with $n$ greater than 50, provided $\epsilon_{r}$ remains constant and Hooke's law applies. This criterion also applies, provided the energy density at rupture for the material remains nearly constant at the impact velocities used for determination of the limiting breaking velocity.

In the example just considered, the breaking velocity for $n=50$ is $42 \mathrm{~m} / \mathrm{sec}$. A tensile impact at this velocity produces a strain pulse of $\epsilon_{0}=1.6$ percent. This suggests a criterion applicable to any textile fiber, for which the energy density at rupture remains nearly constant at high strain rates. For such a fiber a limiting breaking velocity may be found by extrapolation of experimental data taken such that all $v_{n}$ used are less than $0.02 c$, where $c$ is the velocity of propagation of the tensile strain pulse along the sample for the material tested.

\section{Summary and Conclusions}

When the stress-strain characteristics of a textile fiber are measured at longitudinal impact rates exceeding $10 \mathrm{~m} / \mathrm{sec}$ the effects of tension-wave propagation along the fiber must be considered. These initial and reflected waves cause the local strains to vary with time in a stepwise fashion. The average strain in the filament, which is the quantity measured experimentally, does not adequately represent the state of strain under these conditions. As the measured stress is roughly proportional to the local strain at an end, experimental stress-time curves also vary in a stepwise fashion. Stress-strain curves likewise exhibit fluctuations.

The tensile behavior of a Hookean material having a breaking elongation independent of testing speed has been calculated, using a theory in which wave propagation is considered. The results of these calculations have been used to modify the predictions of a simpler theory 
that neglects wave propagation. Thus, it has been shown that a filament weighted at the tail and pulled rapidly at the head at constant speed will attain a maximum average elongation proportional to $\sqrt{n+\frac{1}{2}}$ after the tension wave has been reflected $(\pi / 2) \sqrt{n+\frac{1}{2}}$ times, where $n$ is the ratio of the tail mass to the mass of the specimen.

If $\log$ arithms of the lowest breaking velocities for each $n$ are plotted versus $\log \left(n+\frac{1}{2}\right)$, the theory neglecting wave propagation predicts that a straight line of slope $-\frac{1}{2}$ is obtained, provided that the rupture-energy density is nearly constant at high testing speeds. An extrapolated velocity (for $\log \left(n+\frac{1}{2}\right)$ equals zero) is thus obtained, which is termed the "limiting breaking velocity" of the material; i. e., the velocity above which the sample will always rupture at the head immediately upon impact. Both theories predict the same limiting breaking velocity, provided extrapolation is made from data taken at impact speeds less than 2 percent of the velocity of propagation of a tension-wave pulse along the material. If this limitation is observed, the theory neglecting wave propagation is valid, and extrapolation to the limiting breaking velocity may be made.

\section{Appendix 1. Wave-Theory Solutions}

In table $2, u_{0}$ gives the displacement due to the original incident wave, proceeding toward the tail mass. After reflection at the tail, the displacement, $u$, is computed from the sum of $u_{0}$, and $u_{1}$, the displacement of the reflected wave. Similarly, after the first head reflection, displacement is computed from the sum of $u_{0}, u_{1}$, and $u_{2}$. Displacement due to each reflected wave is a function of a $z$ parameter, which in turn is a function of $x$ and $t$. Only positive values of these $z$ parameters are allowed. Negative values represent position-time events in advance of the wave, where $u=0$.

Velocity functions obtained by differentiating the displacement functions with respect to time are tabulated in table 3. Local-strain functions, obtained by differentiating the displacement functions with respect to the position coordinate along the filament, are given in table 4 .

The method of obtaining these solutions is demonstrated in the following derivation for $u_{1}$.

The initial conditions that must be satisfied for the first reflection at the tail are at $x=-L, \quad t=\frac{L}{c}, \quad z_{0}=0, \quad z_{1}=0$.

Displacement at the tail just before reflection equals the displacement at the tail just after reflection, or $u_{0}(0)=u_{0}(0)+u_{1}(0)$. Hence $u_{1}(0)=0$. Velocity at the tail just before reflection equals the velocity at the tail just after reflection, or $0=v_{0}(0)+v_{1}(0)$. Hence $v_{1}(0)=-v_{0}$. In addition, we have the boundary condition at $x=-L$,

$$
n w \frac{\partial^{2}}{\partial t^{2}}\left[u_{0}\left(z_{0}\right)+u_{1}\left(z_{1}\right)\right]_{x=-L}=E A \frac{\partial}{\partial x}\left[u_{0}\left(z_{0}\right)+u_{1}\left(z_{1}\right)\right]_{x=-L}
$$

or

$$
n w \frac{\partial^{2}}{\partial t^{2}} u_{1}(z)=E A \frac{v_{0}}{c}+E A\left[\frac{\partial}{\partial x} u_{1}\left(z_{1}\right)\right]_{x=-L}
$$

or

$$
n w \frac{d^{2}}{d z^{2}} u_{1}=E A \frac{v_{0}}{c}-\frac{E A}{c} \frac{d}{d z} u_{1}
$$

where $z=t-L / c$, and $A$ is the cross-sectional area of the unstrained filament. If we let

we obtain

$$
\tau=\frac{n w c}{E A}=\frac{n \rho L c}{E}=n \frac{L}{c},
$$

$$
\frac{d^{2} u_{1}}{d z^{2}}+\frac{1}{\tau} \frac{d u_{1}}{d z}=\frac{v_{0}}{\tau}
$$


The solution of this equation that satisfies the initial conditions is

$$
u_{1}=v_{0}\left(t-\frac{L}{c}\right)-2 v_{0} \tau\left[1-e^{-\frac{1}{\tau}\left(t-\frac{L}{c}\right)}\right] .
$$

If we replace $(t-L / c)$ by $z_{1}=t-2(L / c)-x / c$, we obtain the required solution for $u_{1}$.

\section{TABLE 2. Displacement functions}

$$
u=u_{0}+u_{1}+u_{2}+u_{3}+\ldots .
$$

$$
\begin{aligned}
& u_{0}=v_{0} z_{0} \\
& z_{0}=t+\frac{x}{c} \\
& u_{1}=v_{0} z_{1}-2 v_{0} \tau\left[1-e^{-\frac{z_{1}}{\tau}}\right] \text {, where } \tau=n \frac{L}{c} \\
& z_{1}=t-\frac{2 L}{c}-\frac{x}{c} \\
& u_{2}=-v_{0} z_{2}+2 v_{0} \tau\left[1-e^{-\frac{z_{2}}{\tau}}\right] \\
& z_{2}=t-\frac{2 L}{c}+\frac{x}{c} \\
& u_{3}=-v_{0} z_{3}+4 v_{0} \tau\left[1-\left(1+\frac{z_{3}}{\tau}\right) e^{-\frac{z_{3}}{\tau}}\right] \\
& z_{3}=t-\frac{4 L}{c}-\frac{x}{c} \\
& u_{4}=v_{0} z_{4}-4 v_{0} \tau\left[1-\left(1+\frac{z_{4}}{\tau}\right) e^{-\frac{z_{4}}{\tau}}\right] \\
& z_{4}=t-\frac{4 L}{c}+\frac{x}{c} \\
& u_{5}=v_{0} z_{5}-6 v_{0} \tau\left[1-\left(1+\frac{2}{3} \frac{z_{5}}{\tau}+\frac{2}{3} \frac{z_{5}^{2}}{\tau^{2}}\right) e^{-\frac{z_{5}}{\tau}}\right] \\
& z_{5}=t-\frac{6 L}{c}-\frac{x}{c} \\
& u_{6}=-v_{0} z_{6}+6 v_{0} \tau\left[1-\left(1+\frac{2}{3} \frac{z_{6}}{\tau}+\frac{2}{3} \frac{z_{6}^{2}}{\tau^{2}}\right) e^{-\frac{z_{6}}{\tau}}\right] \\
& z_{6}=t-\frac{6 L}{c}+\frac{x}{c} \\
& u_{7}=-v_{0} z_{7}+8 v_{0} \tau\left[1-\left(1+\frac{z_{7}}{\tau}+\frac{1}{3} \frac{z_{7}^{3}}{\tau^{3}}\right) e^{-\frac{z_{7}}{\tau}}\right] \\
& z_{7}=t-\frac{8 L}{c}-\frac{x}{c} \\
& u_{8}=v_{0} z_{8}-8 v_{0} \tau\left[1-\left(1+\frac{z_{8}}{\tau}+\frac{1}{3} \frac{z_{8}^{3}}{\tau^{3}}\right) e^{-\frac{z_{8}}{\tau}}\right] \\
& z_{8}=t-\frac{8 L}{c}+\frac{x}{c} \\
& u_{9}=v_{0} z_{9}-10 v_{0} \tau\left[1-\left(1+\frac{4}{5} \frac{z_{9}}{\tau}+\frac{4}{5} \frac{z_{9}^{2}}{\tau^{2}}-\frac{4}{15} \frac{z_{9}^{3}}{\tau^{3}}+\frac{2}{15} \frac{z_{9}^{4}}{\tau^{4}}\right) e^{-\frac{z_{9}}{\tau}}\right] \\
& z_{9}=t-\frac{10 L}{c}-\frac{x}{c} \\
& u_{10}=-v_{0} z_{10}+10 v_{0} \tau\left[1-\left(1+\frac{4}{5} \frac{z_{10}}{\tau}+\frac{4}{5} \frac{z_{10}^{2}}{\tau^{2}}-\frac{4}{15} \frac{z_{10}^{3}}{\tau^{3}}+\frac{2}{15} \frac{z_{10}^{4}}{\tau^{4}}\right) e^{-\frac{z_{10}}{\tau^{4}}}\right] \\
& z_{10}=t-\frac{10 L}{c}+\frac{x}{c}
\end{aligned}
$$

\section{TABLE 3. Velocity functions}

$$
\begin{aligned}
v & =\frac{\partial u}{\partial t}=\frac{\partial u_{0}}{\partial t}+\frac{\partial u_{1}}{\partial t}+\frac{\partial u_{2}}{\partial t}+\ldots=v_{0}+v_{1}+v_{2}+\ldots \\
v_{0} & =v_{0}\left(\text { for } z_{0}>0\right) \\
v_{1} & =v_{0}-2 v_{0} e^{-\frac{z_{1}}{\tau}} \\
v_{2} & =-v_{0}+2 v_{0} e^{-\frac{z_{2}}{\tau}} \\
v_{3} & =-v_{0}+4 v_{0} \frac{z_{3}}{\tau} e^{-\frac{z_{3}}{\tau}}
\end{aligned}
$$


TABLE 3. Velocity functions-Continued

$$
\begin{aligned}
& v_{4}=v_{0}-4 v_{0} \frac{z_{4}}{\tau} e^{-\frac{z_{4}}{\tau}} \\
& v_{5}=v_{0}-6 v_{0}\left(\frac{1}{3}-\frac{2}{3} \frac{z_{5}}{\tau}+\frac{2}{3} \frac{z_{5}^{2}}{\tau^{2}}\right) e^{-\frac{z_{5}}{\tau}} \\
& v_{6}=-v_{0}+6 v_{0}\left(\frac{1}{3}-\frac{2}{3} \frac{z_{6}}{\tau}+\frac{2}{3} \frac{z_{6}^{2}}{\tau^{2}}\right) e^{-\frac{z_{6}}{\tau}} \\
& v_{7}=-v_{0}+8 v_{0}\left(\frac{z_{7}}{\tau}-\frac{z_{7}^{2}}{\tau^{2}}+\frac{1}{3} \frac{z_{7}^{3}}{\tau^{3}}\right) e^{-\frac{z_{7}}{\tau}} \\
& v_{8}=v_{0}-8 v_{0}\left(\frac{z_{8}}{\tau}-\frac{z_{8}^{2}}{\tau^{2}}+\frac{1}{3} \frac{z_{8}^{3}}{\tau^{3}}\right) e^{-\frac{z_{8}}{\tau}} \\
& v_{9}=v_{0}-10 v_{0}\left(\frac{1}{5}-\frac{4}{5} \frac{z_{9}}{\tau}+\frac{8}{5} \frac{z_{9}^{2}}{\tau^{2}}-\frac{4}{5} \frac{z_{9}^{3}}{\tau^{3}}+\frac{2}{15} \frac{z_{9}^{4}}{\tau^{4}}\right) e^{-\frac{z_{9}}{\tau}} \\
& v_{10}=-v_{0}+10 v_{0}\left(\frac{1}{5}-\frac{4}{5} \frac{z_{10}}{\tau}+\frac{8}{5} \frac{z_{10}^{2}}{\tau^{2}}-\frac{4}{5} \frac{z_{10}^{3}}{\tau^{3}}+\frac{2}{15} \frac{z_{10}^{4}}{\tau^{4}}\right) e^{-\frac{z_{10}}{\tau}}
\end{aligned}
$$

TABLE 4. Local strain functions

$$
\boldsymbol{\epsilon}=\frac{\partial u}{\partial x}=\frac{\partial u_{0}}{\partial x}+\frac{\partial u_{1}}{\partial x}+\ldots=\boldsymbol{\epsilon}_{0}+\boldsymbol{\epsilon}_{1}+\ldots
$$

$\epsilon_{0}=\frac{v_{0}}{c}\left(\right.$ for $\left.z_{0}>0\right)$

$$
\begin{aligned}
& \boldsymbol{\epsilon}_{1}=-\epsilon_{0}+2 \epsilon_{0} e^{-\frac{z_{1}}{\tau}} \\
& \boldsymbol{\epsilon}_{2}=-\epsilon_{0}+2 \epsilon_{0} e^{-\frac{z_{2}}{\tau}} \\
& \boldsymbol{\epsilon}_{3}=\epsilon_{0}-4 \epsilon_{0} \frac{z_{3}}{\tau} e^{-\frac{z_{3}}{\tau}} \\
& \boldsymbol{\epsilon}_{4}=\epsilon_{0}-4 \epsilon_{0} \frac{z_{4}}{\tau} e^{-\frac{z_{4}}{\tau}} \\
& \epsilon_{5}=-\epsilon_{0}+6 \epsilon_{0}\left(\frac{1}{3}-\frac{2}{3} \frac{z_{5}}{\tau}+\frac{2}{3} \frac{z_{5}^{2}}{\tau^{2}}\right) e^{-\frac{z_{5}}{\tau}} \\
& \boldsymbol{\epsilon}_{6}=-\epsilon_{0}+6 \epsilon_{0}\left(\frac{1}{3}-\frac{2}{3} \frac{z_{6}}{\tau}+\frac{2}{3} \frac{z_{6}^{2}}{\tau^{2}}\right) e^{-\frac{z_{6}}{\tau}} \\
& \epsilon_{7}=\epsilon_{0}-8 \epsilon_{0}\left(\frac{z_{7}}{\tau}-\frac{z_{7}^{2}}{\tau^{2}}+\frac{1}{3} \frac{z_{7}^{3}}{\tau^{3}}\right) e^{-\frac{z_{7}}{\tau}} \\
& \epsilon_{9}=-8 \epsilon_{0}\left(\frac{z_{8}}{\tau}-\frac{z_{8}^{2}}{\tau^{2}}+\frac{1}{3} \frac{z_{8}^{3}}{\tau^{3}}\right) e^{-\frac{z_{8}}{\tau}} \\
& \boldsymbol{\epsilon}_{10}=-10 \epsilon_{0}\left(\frac{1}{5}-\frac{4}{5} \frac{z_{9}}{\tau}+\frac{8}{5} \frac{z_{9}^{2}}{\tau^{2}}-\frac{4}{5} \frac{z_{9}^{3}}{\tau^{3}}+\frac{2}{15} \frac{z_{9}^{4}}{\tau^{4}}\right) e^{-\frac{z_{9}}{\tau}} \\
& \epsilon_{0}\left(\frac{1}{5}-\frac{4}{5} \frac{z_{10}}{\tau}+\frac{8}{5} \frac{z_{10}^{2}}{\tau^{2}}-\frac{4}{5} \frac{z_{10}^{3}}{\tau^{3}}+\frac{2}{15} \frac{z_{10}^{4}}{\tau^{4}}\right) e^{-\frac{z_{10}}{\tau}}
\end{aligned}
$$




\section{Appendix 2. Solution, Neglecting Wave Propagation}

Consider a filament of length $L$, cross section $\mathrm{A}$, and density $\rho$ and having a mass $n^{\prime} \rho A L$ attached at the tail, where $n^{\prime}$ is equal to $n+f, n$ is the ratio of the tail mass to the mass of the filament, and $f$ is the fraction of the mass of the filament assumed concentrated at the tail. It is shown in section 4 that the best value for $f$ is $\frac{1}{2}$.

At zero time and subsequently, let the head end of the filament be constrained to move with velocity $v_{0}$. The equation of motion is

$$
n^{\prime} \rho A L \frac{d^{2} u}{d t^{2}}=F,
$$

where $u$ is the displacement of the tail mass, and $F$ is the accelerating force transmitted through the filament to the tail mass.

The strain in the filament, $\epsilon$, is given by

$$
\epsilon=\frac{v_{0} t-u}{L} .
$$

When Hooke's law applies,

$$
F=A E \epsilon,
$$

where $E$ is Young's modulus for the filament material. Substituting (16) and (17) into (15), we obtain

$$
\frac{d^{2} u}{d t^{2}}+\omega_{0}^{2} u=\omega_{0}^{2} v_{0} t,
$$

where

$$
\omega_{0}=\frac{c}{L} \frac{1}{\sqrt{n^{\prime}}},
$$

and

$$
c=\sqrt{\frac{E}{\rho}} .
$$

A solution of (18) satisfying the boundary conditions, $u=0$ and $d u / d t=0$ at $t=0$, is

$$
u=v_{0}\left[t-\frac{1}{\omega_{0}} \sin \omega_{0} t\right]
$$

If we let $t=(L / c) \theta$, and $\epsilon_{0}=v_{0} / c$, we obtain

$$
\begin{aligned}
& u=\epsilon_{0} L\left[\theta-\sqrt{n^{\prime}} \sin \frac{\theta}{\sqrt{n^{\prime}}}\right] \\
& \epsilon=\epsilon_{0} \sqrt{n^{\prime}} \sin \frac{\theta}{\sqrt{n^{\prime}}} .
\end{aligned}
$$

In a previous paper (see footnote 2 ) the following equation was derived:

$$
\log v_{n}=-\frac{1}{2} \log \frac{\left(n+\frac{1}{2}\right)\left(m+\frac{1}{2}\right)}{n+m+1}+\log \sqrt{\frac{2}{\rho} \int_{0}^{\epsilon_{r}} \sigma d \epsilon,}
$$

where $v_{n}$ is the lowest impact velocity sufficient to cause rupture in a filament to which a tail mass $n$ times that of the filament and a head mass $m$ times that of the filament, are attached. For comparison with the work discussed here, we let $m \rightarrow \infty$, obtaining

$$
\log v_{n}=-\frac{1}{2} \log \left(n+\frac{1}{2}\right)+\log \sqrt{\frac{2}{\rho} \int_{0}^{\epsilon_{r}} \sigma d \epsilon} .
$$

Washington, December 31, 1954. 